Pacific Journal of Mathematic 


\title{
BOUNDED APPROXIMATION BY RATIONAL FUNCTIONS
}

\author{
STEPhen Fisher
}

Let $D$ be a bounded open subset of the complex plane $\leftarrow$ which is the interior of its closure, and let $h$ be a bounded analytic function on $D$. The classical theorem of Runge implies that there is a sequence of rational functions with poles in the complement of the closure of $D$ which converges to $h$ uniformly on compact subsets of $D$. The question naturally arises as whether this sequence may be chosen so that the supremum norms over $D$ of the rational functions remain uniformly bounded. Of course, if the boundary of $D$ consists of a finite number of disjoint circles (that is, $D$ is a circle domain), then it is a classical result that the approximating sequence may be chosen so that their norms do not exceed the norm of $h$. But suppose that the boundary of $D$ is quite complicated or $D$ has infinitely many components in its complement. This general question has been the subject of several recent papers and is the subject of this one.

In [4] Rubel and Shields showed that if the complement of the closure of $D$ is connected, then there is a sequence $\left\{h_{n}\right\}$ of polynomials with $\left\|h_{n}\right\| \leqq\|h\|$ and $h_{n}(z) \rightarrow h(z)$ for each $z$ in $D$. Ahern and Sarason extended this result in [2] and proved that if a bounded open set $D$ is the interior of its closure and has only finitely many components in its complement, then such bounded pointwise approximation is always possible, where the approximating functions have poles in the (finitely many) components of the complement of the closure of $D$, and their norms on $D$ do not exceed the norm of the limit function.

The chief results in this paper show that rather elementary techniques may be used to extend the theorems of Rubel-Shields and Ahern-Sarason to certain domains with infinitely many complementary components.

We first introduct some notation to be used throughout the remainder of the paper: $U$ is the open unit disc, $\{z|| z \mid<1\} ; \Gamma$ is the unit circle, $\{z|| z \mid=1\}$; if $D$ is an open set, $H^{\infty}(D)$ is the space of bounded analytic functions on $D$ and $\bar{D}$ denotes the closure of $D$; if $K$ is a compact set, then $R(K)$ is the uniform closure on $K$ of the rational functions with poles off $K$; finally, $\partial S$ denotes the boundary of $S$.

THEOREM 1. Let $S_{1}, S_{2}, \ldots$ be a sequence of disjoint closed discs in the open unit disc $U$ which are centered on the positive real axis 
and whose centers, $c_{n}$, and radii, $r_{n}$, decrease to 0 . Suppose that there is a constant $\delta>1$ such that $\left(c_{n}-r_{n}\right) \geqq \delta\left(c_{n+1}+r_{n+1}\right)$ for all n. Let $D$ be the domain $U-\{0\} \cup\left[\bigcup_{i=1}^{\infty} S_{i}\right]$. If $f \in H^{\infty}(D)$, then there is a sequence $\left\{f_{n}\right\}$ of rational functions with poles off $\bar{D}$ such that $\left\|f_{n}\right\| \leqq\{\delta /(\delta-1)\}\|f\|$ and $f_{n}(z) \rightarrow f(z)$ for each $z$ in $D$.

Proof. Let $\gamma_{n}$ be the circle of radius $c_{n}+r_{n}$ about 0 and let $D_{n}=U-S_{1} \cup \cdots \cup S_{n-1}$. For $z$ in $D_{n}$ define

$$
f_{n}(z)=\sum_{j=0}^{n-1} \frac{1}{2 \pi i} \int_{\Gamma_{j}} \frac{f^{*}(w)}{w-z} d w
$$

where $\Gamma_{0}$ is the unit circle, $\Gamma_{i}=\partial S_{i}$ for $1 \leqq i \leqq n-1$ and the $f^{*}$ on $\partial D_{n}$ is the usual boundary-value function of $f$. For $z$ near $\partial D_{n}$ we have

$$
f(z)=f_{n}(z)+\frac{1}{2 \pi i} \int_{r_{n}} \frac{f(w)}{w-z} d w
$$

by Cauchy's formula. Thus, for $z$ near $\partial D_{n}$,

$$
\left|f_{n}(z)\right| \leqq|f(z)|+\left|\frac{1}{2 \pi i} \int_{r_{n}} \frac{f(w)}{w-z} d w\right|
$$

and hence

$$
\left\|f_{n}\right\| \leqq\|f\|\left[1+\frac{1}{2 \pi} \frac{2 \pi\left(r_{n}+c_{n}\right)}{\left(c_{n-1}-r_{n-1}\right)-\left(r_{n}+c_{n}\right)}\right] \leqq\|f\|\left[\frac{\delta}{\delta-1}\right]
$$

as desired. It is immediate that $f_{n}(z) \rightarrow f(z)$ for each $z$ in $D$ since $c_{n}+r_{n} \rightarrow 0$ as $n \rightarrow \infty$; thus $f_{n} \rightarrow f$ uniformly on compact subsets of $D$. Since $D_{n}$ is a circle domain, the $f_{n}$ 's may be replaced by rational functions with poles off $\bar{D}$ without increasing the estimate on the norms and without affecting the uniform convergence on compact subsets.

THEOREM 2. Let I be an arbitrary closed subset of the interval $\left[-\frac{1}{2}, \frac{1}{2}\right]$ of zero arc length. Let $\left\{c_{i}\right\}$ be a countable set of distinct points in the unit disc with $\operatorname{Im} c_{i} \neq 0$ such that $I$ is precisely the set of accumulation points of $\left\{c_{i}\right\}$. Let $S_{i}$ be a closed disc in $U-I$ centered at $c_{i}$ of radius $r_{i}$ where the radii are chosen so small that (a) $S_{i} \cap S_{j}=\varnothing$ for $i \neq j$ and (b) $\sum_{i=1}^{\infty} r_{i} / d_{i}=C<\infty$ where $d_{i}$ is the distance from the $i^{\text {th }}$ disc to the nearest (other) disc. Let $D=U-$ $I \cup \bigcup_{i=1}^{\infty} S_{i}$. If $f \in H^{\infty}(D)$, then there is a sequence $\left\{f_{n}\right\}$ of elements of $R(\bar{D})$ such that $\left\|f_{n}\right\| \leqq(C+1)\|f\|$ and $f_{n}(z) \rightarrow f(z)$ for each $z$ in $D$. 
Proof. Let $E_{n}=U-S_{1} \cup \cdots \cup S_{n}$ and for $z$ in $E_{n}$ define

$$
f_{n}(z)=\frac{1}{2 \pi i} \int_{\Gamma} \frac{f^{*}(w)}{w-z} d w+\sum_{j=1}^{n} \frac{1}{2 \pi i} \int_{\partial s_{j}} \frac{f^{*}(w)}{w-z} d w
$$

where the $f^{*}$ in the integrand is the usual boundary-value function for $f$. Cauchy's formula holds in $D$ in the following form

$$
f(z)=\frac{1}{2 \pi i} \int_{\Gamma} \frac{f^{*}(w)}{w-z} d w+\sum_{j=1}^{\infty} \frac{1}{2 \pi i} \int_{\partial S_{j}} \frac{f^{*}(w)}{w-z} d w
$$

for each $z$ in $D$. This is easily proved in the following manner. For each positive integer $n$ choose a finite number of open discs whose union, $U_{n}$, contains $I$ and the sum of whose radii is less than $n^{-1}$. This is possible since $I$ has zero linear measure. Let $D_{n}=D-\bar{U}_{n}$. Then the boundary of $D_{n}$ is rectifiable and consists of a finite number of piecewise smooth curves. If $\gamma_{n}$ denotes the boundary of $U_{n}$, and $X_{n}$ the intersection of $\partial D_{n}$ with $\partial D$, we have by Cauchy's formula

$$
f(z)=\frac{1}{2 \pi i} \int_{X_{n}} \frac{f^{*}(w)}{w-z} d w+\frac{1}{2 \pi i} \int_{r_{n}} \frac{f(w)}{w-z} d w .
$$

The second integral is bounded by $C\|f\|_{\infty} n^{-1}$ where $C$ is a constant depending only on the distance between $z$ and $U_{n}$. The first integral differs from the integral in $\left(^{*}\right)$ by less than a constant times the tail of the convergent series $\sum_{j=1}^{\infty} r_{j} / d_{j}$. Letting $n$ approach infinity we obtain the desired conclusion. From this form of Cauchy's formula estimates like those in Theorem 1 show that $\left\|f_{n}\right\| \leqq(1+C)\|f\|$ and that $f_{n}(z) \rightarrow f(z)$ for each $z$ in $D$. Use the fact that $E_{n}$ is a circle domain to replace the $f_{n}$ 's by elements of $R(\bar{D})$.

A Counter-example. Bounded approximation of $H^{\infty}$ functions by rational functions is not possible if the set $I$ of accumulation points of the complementary components is "too" big, no matter how nice these components are.

Let $I$ be an arbitrary closed subset of $\left[-\frac{1}{2}, \frac{1}{2}\right]$ of positive arclength and let $\left\{S_{i}\right\}$ be a sequence of disjoint closed discs in $U-I$, the sum of whose radii is finite, which collect at each point of $I$ and only there. Then there are bounded analytic functions on $D=U-$ $I \cup \bigcup_{i=1}^{\infty} S_{i}$ which cannot be approximated pointwise on $D$ by a uniform$l y$ bounded sequence of rational functions.

To see this, we note that since $I$ has positive length there is a function $h$ which is bounded, nontrivial, and analytic on the complement of $I$ relative to the sphere [1; p. 254]. Without loss of generality it may be assumed that 


$$
h^{\prime}(\infty)=\frac{1}{2 \pi i} \int_{\Gamma} h(z) d z \neq 0
$$

Since $h$ is analytic on a neighborhood of $S_{i}, \int_{\partial S_{i}} h(z) d z=0$ for $i=1,2, \ldots$ Note that for $\varphi$ in $R(\bar{D})$, we have $\int_{X} \varphi(z) d z=0$ where $X=\partial D-I$ and $X$ has the usual positive orientation.

Suppose $\left\{\varphi_{n}\right\}$ is a sequence of elements of $R(\bar{D})$ such that $\left\|\varphi_{n}\right\| \leqq$ $M$ for some $M$ and $\varphi_{n}(z) \rightarrow h(z)$ for each $z$ in $D$. Then at least some subsequence of $\left\{\varphi_{n}\right\}$ converges to $h$ in the weak-star topology of $L^{\infty}(X, d z)$. But then

$$
0=\int_{X} \varphi_{n} d z \rightarrow \int_{X} h d z=\int_{\Gamma} h d z=2 \pi i h^{\prime}(\infty) \neq 0,
$$

a contradiction.

THEOREM 3. Let $\left\{S_{i}\right\}$ be a sequence of pairwise disjoint closed discs in $U$ all of whose accumulation points form a closed set $E$ in unit the circle of zero arc length. Let $D=U-\bigcup_{i=1}^{\infty} S_{i}$. Suppose that for each $i$ there is a point $p_{i}$ in $S_{i}$ such that $\sum_{i=1}^{\infty}\left(1-\left|p_{i}\right|\right)<\infty$.

If $h \in H^{\infty}(D)$, then there is a sequence $\left\{h_{n}\right\}$ of elements of $R(\bar{D})$ such that $\left\|h_{n}\right\| \leqq\|h\|$ and $h_{n}(z) \rightarrow h(z)$ for each $z$ in $D$.

The proof of the theorem will require the following lemma. The lemma involves harmonic measure, details about which may be found in [3].

Lemma. Let $D$ be the domain of Theorem 3. If $f$ is a bounded harmonic function on $D$, then there is a unique function $F$ in $L^{\infty}(\partial D, \mu)$, where $\mu$ is harmonic measure for some point $p$ of $D$, such that $f$ is the harmonic extension to $D$ of $F$ and $\|f\|_{\infty}=\|F\|_{\infty}$. Further, $f \geqq 0$ implies that $F \geqq 0$.

Proof of the lemma. We only sketch the proof here since it is a simple limiting argument. Let $\left\{D_{n}\right\}$ be a sequence of subdomains of $D$ satisfying the following three conditions: (a) $D_{n} \subset D_{n+1}$ and $\cup D_{n}=D$; (b) $D_{n}$ is the interior of its closure and the complement of $D_{n}$ has only a finite number of components; (c) $\bigcup_{n=1}^{\infty} \partial D \cap \partial D_{n}=$ $\partial D-E$. The restriction of $f$ to $D_{n}$ is a bounded harmonic function on $D_{n}$. Since the conclusions of the lemma are known for $D_{n}$ (see [2; §3]), there is a unique bounded function $F_{n}$ in $L^{\infty}\left(\partial D_{n}, \mu_{n}\right)$ such that the harmonic extension of $F_{n}$ to $D_{n}$ equals $f$ and

$$
\left\|F_{n}\right\|_{\infty}=\left\|f \mid D_{n}\right\| \leqq\|f\| \text {. }
$$


Hence, $F_{n}=F_{m}$ on $\partial D \cap \partial D_{m}$ for all $n \geqq m$. This common function on $\partial D-E$ is easily shown to be the desired element of $L^{\infty}(\partial D, \mu)$.

Now we turn to the proof of Theorem 3 .

We suppose first that $1 \leqq|h(z)| \leqq 3$ for all $z$ in $D$ and hence that $h(z)=\exp \left[f(z)+i f^{*}(z)\right]$ where $f$ is a positive bounded harmonic function on $D$ and $f^{*}$ is the harmonic conjugate of $f$.

By the lemma there is a nonnegative function $F$ in $L^{\infty}(\partial D, \mu)$ with $f(z)=\int_{\partial D} F d \mu_{z}$ for all $z$ in $D$ and $\|F\|_{\infty}=\|f\|_{D} \leqq 2$. Let $D_{n}=$ $U-\bigcup_{i=1}^{n} S_{i}$ and let $f_{n}$ be the harmonic extension to $D_{n}$ of $F \mid \partial D_{n}$. Then $\left\|f_{n}\right\| \leqq 2$ and $f_{n} \rightarrow f$ uniformly on compact subsets of $D$.

Let $f_{n}^{*}$ be the harmonic conjugate of $f_{n}$ on $D_{n}$, and let $p_{n j}$ be the period of $f_{n}^{*}$ about $S_{j}, 1 \leqq j \leqq n$. $h$ is singlevalued so that the period of $f^{*}$ about each $S_{j}$ is some integer multiple of $2 \pi$. Since $f_{n}$ converges uniformly on compact subsets of $D$ to $f, p_{n j} \rightarrow 0(\bmod 2 \pi)$ as $n \rightarrow \infty$ for each $j=1,2, \cdots$.

For fixed $j$, let $r_{n j}$ be the unique number in $\left[\frac{1}{2}, \frac{3}{2}\right)$ such that $2 \pi r_{n j}=p_{n j}(\bmod 2 \pi)$. Since $p_{n j} \rightarrow 0(\bmod 2 \pi)$ for fixed $j$, we have $r_{n j} \rightarrow 1$ for fixed $j$ as $n \rightarrow \infty$. Define a sequence of negative harmonic functions as follows: let

$$
a_{n}(z)=\sum_{j=1}^{n} r_{n j} \log \left|\frac{z-p_{j}}{1-\bar{p}_{j} z}\right|
$$

for $z$ in $D_{n}$. Then because $\sum\left(1-\left|p_{j}\right|\right)<\infty$, we have

$$
a_{n}(z) \rightarrow \sum_{j=1}^{\infty} \log \left|\frac{z-p_{j}}{1-\bar{p}_{j} z}\right|
$$

for each $z$ in $D$ and the convergence is uniform on compact subsets. Let $g_{n}=\exp \left[f_{n}+a_{n}+i\left(f_{n}^{*}+a_{n}^{*}\right)\right]$. Then $g_{n}$ is a single-valued analytic function on $D_{n}$ and for $z$ in $D, g_{n}(z) \rightarrow h(z) B(z)$ where $B$ is the Blaschke product on $U$ whose zeros are at the points $\left\{p_{j}\right\}$.

Now if $h \in H^{\infty}(D)$ and $\|h\| \leqq 1$, then $2+h$ is in $H^{\infty}(D)$ and $1 \leqq$ $|h(z)+2| \leqq 3$. Hence, there is a sequence $\left\{g_{n}\right\}$ with $g_{n} \in H^{\infty}\left(D_{n}\right)$ such that $\left\|g_{n}\right\| \leqq\|h\|+2$ and $g_{n}$ converges pointwise on $D$ to $(2+h) B$. Hence, if $h_{n}=g_{n}-2 B$, then $h \in H^{\infty}\left(D_{n}\right),\left\|h_{n}\right\| \leqq\|h\|+4$ and $h_{n}(z) \rightarrow$ $h(z) B(z)$ for all $z$ in $D$. Thus, $h_{n}$ converges to $h B$ uniformly on compact subsets of $D$. Since $D_{n}$ is a circle domain, we may conclude that for each $h$ in $H^{\infty}(D)$ there is a uniformly bounded sequence $\left\{h_{n}\right\}$ of elements of $R(\bar{D})$ such that $h_{n}(z) \rightarrow h(z) B(z)$ for each $z$ in $D$. It will be shown later that this implies that the $h_{n}$ 's may actually be chosen so that $\left\|h_{n}\right\|_{D} \leqq\|h B\|_{D}$. (See Theorem 4 and the observations that follow it.) Assuming this, we may complete the proof of the theorem. Let 


$$
B_{n}(z)=\prod_{n+1}^{\infty} \frac{p_{j}-z}{1-\bar{p}_{j} z}\left(\frac{\bar{p}_{j}}{p_{j}}\right)
$$

for $z$ in $U$ and let

$$
C_{n}=\prod_{1}^{n} \frac{p_{j}-z}{1-\bar{p}_{j} z}\left(\frac{\bar{p}_{j}}{p_{j}}\right)
$$

for $z$ in $U$. Let $h$ be in $H^{\infty}(D)$. Since the interior of $S_{j}$ is dense in $S_{j}$ we may assume without any loss of generality that $p_{j}$ lies in the interior of $S_{j}$ for each $j$. Then $h / C_{n}$ is in $H^{\infty}(D)$. Note that $h B_{n}$ converges uniformly on compact subsets of $D$ to $h$ as $n \rightarrow \infty$. Since each $h B_{n}=\left(h / C_{n}\right)(B)$ may be approximated uniformly on compact subsets of $D$ by elements of $R(\bar{D})$ whose norms do not exceed $\left\|h B_{n}\right\| \leqq$ $\|h\|$, the same is true for $h$.

Theorem 4. Let $D$ be the domain of Theorem 3 and let $\mu$ be harmonic measure for some point $p$ in $D$. If $f$ lies in the weakstar closure of $R(\bar{D})$ in $L^{\infty}(\partial D, \mu)$, then there is a sequence $\left\{f_{n}\right\}$ of elements of $R(\bar{D})$ such that $\left\|f_{n}\right\| \leqq\|f\|$ and $f_{n} \rightarrow f$ in the weak-star topology of $L^{\infty}$.

Proof. Note that if $F$ is a closed subset of arc length zero in $\Gamma$ or in $\partial S_{i}$ for some $i$, then there is an element $g$ of $R(\bar{D})$ such that $g=1$ on $F$ and $|g|<1$ on $\partial D-F$. This follows immediately from the fact that closed sets of arc length zero on the unit circle are peak sets for closure of the polynomials on the closed unit disc. Note also that arc length and harmonic measure are mutually absolutely continuous on on $\partial D$. Thus if $m$ is a measure on $\partial D$ which annihilates $R(\bar{D})$, then $m$ is absolutely continuous with respect to $\mu$.

The remainder of the proof now parallels that of [2, Th. 3] and hence need not be repeated.

Theorem 4 implies the following: let $D$ be the domain of Theorem 3 and let $f \in H^{\infty}(D)$. If there is some uniformly bounded sequence $\left\{f_{n}\right\}$ of elements of $R(\bar{D})$ such that $f_{n}(z) \rightarrow f(z)$ for all $z$ in $D$, then the $f_{n}$ may be chosen so that $\left\|f_{n}\right\| \leqq\|f\|$. This follows from the lemma and Theorem 4 as follows.

By the lemma there is a unique element $F$ of $L^{\infty}(\partial D, \mu)$ such that $f(z)=\int_{\partial D} F d \mu_{z}$ for every $z$ in $D$ and $\|f\|=\|F\|$. The functions $f_{n}$ are continuous on $\partial D$ and uniformly bounded there. Hence, they have a weak-star convergent subsequence in $L^{\infty}$, which we again denote by $f_{n}$. If $g$ is the limit of this sequence, then $f(z)=\int_{\partial D} g d \mu_{z}$ because

$$
f(z)=\lim f_{n}(z)=\lim \int_{\partial D} f_{n} d \mu_{z}=\int_{\partial D} g d \mu_{z} .
$$


Hence, $g=F$ a.e. $\mu$ and thus $F$ lies in the weak-star closure of $R(\bar{D})$ in $L^{\infty}$. But now Theorem 4 implies there is a sequence $g_{n}$ in $R(\bar{D})$ with $\left\|g_{n}\right\| \leqq\|F\|=\|f\|$ and $g_{n} \rightarrow F$ in the weak-star topology of $L^{\infty}$. Whence,

$$
g_{n}(z)=\int_{\partial D} g_{n} d \mu_{z} \rightarrow \int_{\partial D} F d \mu_{z}=f(z)
$$

for each $z$ in $D$ because the harmonic measures are mutually absolutely continuous. This establishes the conclusion.

Final Remarks. The conclusions of Theorems 1, 2, and 3 do not depend on the smoothness of the boundary. Thus, for example, instead of deleting closed discs from $U$ to form $D$ in Theorem 3, the deleted sets $S_{i}$ may be any compact, pairwise disjoint subsets of $U$ provided that (a) the interior of each $S_{i}$ is connected and dense in $S_{i}$ and the complement of each $S_{i}$ is connected and (b) the set of accumulation points of the $S_{i}$ forms a closed set $E$ in $\Gamma$ of zero arc length and there is a point $p_{i}$ in each $S_{i}$ such that $\sum_{i=1}^{\infty}\left(1-\left|p_{i}\right|\right)<\infty$. The proof given is easily modified to include this more general case. Similar comments apply to the lemma and Theorm 4 (which are needed to prove Theorem 3) and to Theorems 1 and 2.

Finally we note that if $D$ is a domain which satisfies the hypotheses of either Theorem 1 or Theorem 3, then each function continuous on $\bar{D}$ and analytic on $D$ may be uniformly approximated on $\bar{D}$ by a sequence of rational functions with poles off $\bar{D}$. This follows readily from the fact that $H^{\infty}(D)$ may be considered to be the weak-star closure of $R(\bar{D})$ in $L^{\infty}(\partial D, m)$ where $m$ is either harmonic measure in the case of the domain of Theorem 3 or $m$ is harmonic measure plus a point mass at the origin in the case that $D$ is the domain of Theorem 1 , and the fact that any measure on $\partial D$ which annihilates $R(\bar{D})$ is absolutely continuous with respect to $m$.

\section{REFERENCES}

1. L. Ahlfors and L. Sario, Riemann surfaces, Princeton University Press, Princeton, 1960.

2. P. R. Ahern and D. Sarason, On some hypo-Dirichlet algebras of analytic functions, Amer. J. Math. 89 (1967), 932-941.

3. T. W. Gamelin and H. Rossi, "Jensen measures and algebras of analytic functions"; Function algebras, F. T. Birtel, ed; Chicago, Scott Foresman and Co., 1966.

4. L. A. Rubel and A. L. Shields, Bounded approximation by polynomials, Acta Mathematica 112 (1964).

Received January 19, 1968. This paper constitutes a portion of the author's doctoral thesis written under the direction of Professor Frank Forelli, submitted at 
the University of Wisconsin in June, 1967. Research partially supported by NSF Grant GP 8660.

The author would like to thank Professor Forelli for his patience and advice. UNIVERSITY OF WISCONSIN AND

Massachusetts Institute of Technology 


\section{PACIFIC JOURNAL OF MATHEMATICS}

\section{EDITORS}

H. ROYDEN

Stanford University

Stanford, California

\section{R. $R$ PHELPS}

University of Washington

Seattle, Washington 98105

\section{J. DugunduI}

Department of Mathematics

University of Southern California

Los Angeles, California 90007

RICHARD ARENS

University of California

Los Angeles, California 90024

\section{ASSOCIATE EDITORS}

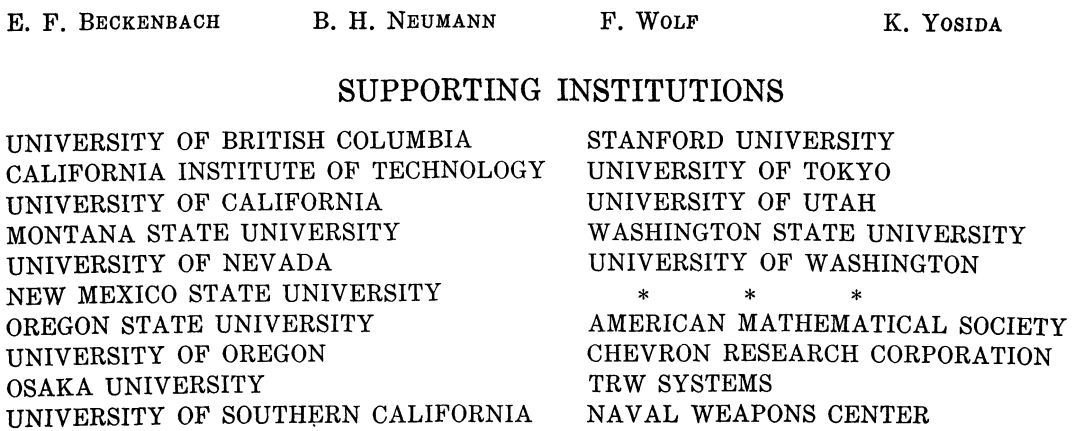

The Supporting Institutions listed above contribute to the cost of publication of this Journal, but they are not owners or publishers and have no responsibility for its content or policies.

Mathematical papers intended for publication in the Pacific Journal of Mathematics should be in typed form or offset-reproduced, double spaced with large margins. Underline Greek letters in red, German in green, and script in blue. The first paragraph or two must be capable of being used separately as a synopsis of the entire paper. It should not contain references to the bibliography. Manuscripts, in duplicate if possible, may be sent to any one of the four editors. Please classify according to the scheme of Math. Rev. 36, 1539-1546. All other communications to the editors should be addressed to the managing editor, Richard Arens, University of California, Los Angeles, California, 90024.

50 reprints are provided free for each article; additional copies may be obtained at cost in multiples of 50 .

The Pacific Journal of Mathematics is published monthly. Effective with Volume 16 the price per volume (3 numbers) is $\$ 8.00$; single issues, $\$ 3.00$. Special price for current issues to individual faculty members of supporting institutions and to individual members of the American Mathematical Society: $\$ 4.00$ per volume; single issues $\$ 1.50$. Back numbers are available.

Subscriptions, orders for back numbers, and changes of address should be sent to Pacific Journal of Mathematics, 103 Highland Boulevard, Berkeley, California, 94708.

PUBLISHED BY PACIFIC JOURNAL OF MATHEMATICS, A NON-PROFIT CORPORATION

Printed at Kokusai Bunken Insatsusha (International Academic Printing Co., Ltd.), 7-17, Fujimi 2-chome, Chiyoda-ku, Tokyo, Japan. 


\section{Pacific Journal of Mathematics}

Vol. 28, No. $2 \quad$ April, 1969

Richard Arens and Donald George Babbitt, The geometry of relativistic

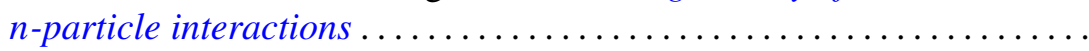

Kirby Alan Baker, Hypotopological spaces and their embeddings in lattices

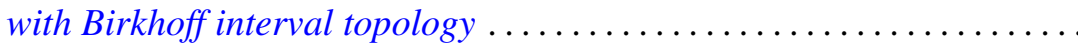

J. Lennart (John) Berggren, Finite groups in which every element is conjugate to its inverse ........................... 289

Beverly L. Brechner, Homeomorphism groups of dendrons . . . . . . . . . . . 295

Robert Ray Colby and Edgar Andrews Rutter, QF - 3 rings with zero singular ideal ................................. 303

Stephen Daniel Comer, Classes without the amalgamation property....... 309

Stephen D. Fisher, Bounded approximation by rational functions ......... 319

Robert Gaines, Continuous dependence for two-point boundary value problems..................................... 327

Bernard Russel Gelbaum, Banach algebra bundles ............... 337

Moses Glasner and Richard Emanuel Katz, Function-theoretic degeneracy criteria for Riemannian manifolds ...................... 351

Fletcher Gross, Fixed-point-free operator groups of order $8 \ldots \ldots \ldots \ldots 357$

Sav Roman Harasymiv, On approximation by dilations of distributions . . . . 363

Cheong Seng Hoo, Nilpotency class of a map and Stasheff's criterion ... . . 375

Richard Emanuel Katz, A note on extremal length and modutus.......... 381

H. L. Krall and I. M. Sheffer, Difference equations for some orthogonal polynomials ................................

Yu-Lee Lee, On the construction of lower radical properties ........... 393

Robert Phillips, Liouville's theorem........................... 397

Yum-Tong Siu, Analytic sheaf cohomology groups of dimension $n$ of

n-dimensional noncompact complex manifolds ..... . .

Michael Samuel Skaff, Vector valued Orlicz spaces. II...

James DeWitt Stein, Homomorphisms of $B^{*}$-algebras .... . .

Mark Lawrence Teply, Torsionfree injective modules .... . . .

Richard R. Tucker, The $\delta^{2}$-process and related topics. II .

David William Walkup and Roger Jean-Baptiste Robert Wets, Lifting

projections of convex polyhedra...

Thomas Paul Whaley, Large sublattices of a lattice. 